\title{
The Importance of History for Philosophy of Psychiatry: The Case of the DSM and Psychiatric Classification
}

\author{
Jonathan Y. Tsou \\ Iowa State University \\ Department of Philosophy and Religious Studies \\ jtsou@iastate.edu
}

\begin{abstract}
Recently, some philosophers of psychiatry (viz., Rachel Cooper and Dominic Murphy) have analyzed the issue of psychiatric classification. This paper expands upon these analyses and seeks to demonstrate that a consideration of the history of the Diagnostic and Statistical Manual of Mental Disorders (DSM) can provide a rich and informative philosophical perspective for critically examining the issue of psychiatric classification. This case is intended to demonstrate the importance of history for philosophy of psychiatry, and more generally, the potential benefits of historically-informed approaches to philosophy of science.
\end{abstract}

\section{Keywords}

philosophy of psychiatry, psychiatric classification, the Diagnostic and Statistical Manual of Mental Disorders

Forthcoming in Journal of the Philosophy of History 


\section{Introduction}

In recent years, some philosophers of science have been interested in questions concerning the classification of mental disorders. The aim of this paper is to qualify and expand upon these analyses by taking into account relevant aspects of the history of psychiatry. In engaging in such a task, I aim to show how a consideration of the history of the Diagnostic and Statistical Manual of Mental Disorders (DSM) can provide a rich and expansive perspective for addressing philosophical issues concerning psychiatric classification. In this regard, the paper seeks to illustrate the important methodological role that history can play in the context of philosophy of psychiatry.

The paper proceeds as follows. I begin by reviewing the philosophical arguments concerning psychiatric classification offered by Rachel Cooper and Dominic Murphy. In particular, I review Cooper's argument that the DSM's goal of classifying natural kinds is unlikely to be achieved in practice because of external pressures on the manual, and Murphy's argument that psychiatry ought to adopt a causal approach to classification. I subsequently examine the history of psychiatric classification embedded in the history of the DSM in the latter half of the twentieth century in order to show how Cooper's and Murphy's arguments can be qualified. My examination of history focuses on American psychiatry's "revolution in classification" - associated with the publication of DSM-III in 1980 - in which the DSM moved away from a psychoanalytic and etiological approach (characteristic of DSM-I and DSM-II) to an "atheoretical” and descriptive approach. I present the historical factors responsible for this shift in terms of the crisis that psychoanalytically-oriented psychiatry faced in the 1960s and 1970s, and the crucial role of the scientifically-oriented "neo-Kraepelinian" task force led by Robert Spitzer that was responsible for revising DSM-II. I argue that the history of the DSM highlights psychiatry's distinctive status as an applied and practically-oriented science, and that the philosophical analyses of psychiatric classification offered by Cooper and Murphy fail to sufficiently appreciate this historical fact. From this standpoint, I offer some qualifications and amendments to Cooper's and Murphy's arguments.

\section{Philosophical Arguments about Psychiatric Classification}

Rachel Cooper and Dominic Murphy have recently offered provocative and interesting philosophical arguments concerning psychiatric classification. ${ }^{1}$ Both Cooper's and Murphy's arguments are presented with reference to the Diagnostic and Statistical Manual of Mental Disorders (DSM), which is the most influential and widely used classification manual in the context of contemporary mental health discourse and practices. ${ }^{2}$ Cooper argues that the DSM's

\footnotetext{
${ }^{1}$ R. Cooper, Classifying Madness: A Philosophical Examination of the Diagnostic and Statistical Manual of Mental Disorders (Dordrecht: Springer, 2005); D. Murphy, Psychiatry in the Scientific Image (Cambridge, MA: MIT Press, 2006).

${ }^{2}$ The DSM has been published regularly by the American Psychiatric Association (APA) since 1952, and is currently in its fourth edition. Six editions of the DSM have been published: (1) Diagnostic and Statistical Manual: Mental Disorders (Washington, DC: American Psychiatric Association, 1952); (2) Diagnostic and Statistical Manual of Mental Disorders, $2^{\text {nd }}$ ed. (Washington, DC: American Psychiatric Association, 1968); (3) Diagnostic and Statistical Manual of Mental Disorders, $3^{\text {rd }}$ ed. (Washington, DC: American Psychiatric Association, 1980); (4) Diagnostic and Statistical Manual of Mental Disorders, $3^{\text {rd }}$ ed., Revised (Washington, DC: American Psychiatric Association, 1987); (5) Diagnostic and Statistical Manual of Mental Disorders, $4^{\text {th }}$ ed. (Washington, DC: American
} 
aim of classifying mental disorders as natural kinds is feasible; however, she suggests that this ideal is unlikely to be achieved because of external pressures on the manual. Murphy argues that the descriptive approach to classification favored by the DSM since the publication of DSM-III should be abandoned in favor of a causal approach to psychiatric classification.

\subsection{Cooper on the Limitations of Classifying Natural Kinds}

Cooper's argument concerns the prospects of classifying mental disorders as natural kinds, and she suggests that the likelihood of achieving such a goal is gloomy. ${ }^{3}$ Cooper maintains that some mental disorders are natural kinds insofar as members of a kind share similar key properties (e.g., a similar genetic basis) that determine other salient characteristics of the disorder. She suggests that the DSM is unlikely to classify disorders as natural kinds because of the way that the manual is influenced by social and financial factors (e.g., the medical insurance industry). ${ }^{4}$

Cooper defends a non-essentialist account of natural kinds that stipulates that members of a natural kind are objectively similar in theoretically important ways. As she puts it, "members of a natural kind all possess similar determining properties, where the determining properties of an entity are those properties that determine its other properties". ${ }^{5}$ On this account, determining properties are the key properties of an entity that render members within a class of entity similar. More specifically, determining properties are properties that are of particular interest to scientists, and appear in natural laws that support inductive inferences about members of a kind. For example, the determining property of Huntington's Chorea is an anomaly on chromosome four, which causes the appearance of its characteristic symptoms (i.e., jerky movements, progressive motor dysfunction, cognitive impairments, memory deficits, dementia). In this sense, Cooper maintains that mental disorders that are natural kinds possess similar determining properties (e.g., a genetic or neurobiological anomaly). From this standpoint, a manual such as the DSM could classify mental disorders as natural kinds insofar as psychiatrists could use empirical data to identify categories for grouping together classes that possess similar determining properties (e.g., grouping together disorders that involve a similar neurotransmitter dysfunction).

Cooper argues that the DSM's goal of classifying mental disorders in a naturalistic manner is unlikely to be achieved in practice because of various social and financial factors that shape and constrain the organization of the DSM. Specifically, she argues that pressures from the pharmaceutical and medical insurance industries have functioned to hinder the goal of classifying natural kinds. With respect to the pharmaceutical industry, Cooper brings attention to

\footnotetext{
Psychiatric Association, 1994); and (6) Diagnostic and Statistical Manual of Mental Disorders, $4^{\text {th }}$ ed., Text Revision (Washington, DC: American Psychiatric Association, 2000). For convenience, I refer to the various editions of the DSM as DSM-I (1952), DSM-II (1968), DSM-III (1980), DSM-III-R (1987), DSM-IV (1994), and DSM-IV-TR (2000); at the time of writing this paper, DSM-V is in preparation.

${ }^{3}$ See Cooper, Classifying Madness, ch. 4; R. Cooper, "What is Wrong with the DSM", History of Psychiatry 15 (2004), 5-25.

${ }^{4}$ Cooper also argues that the goal of classifying mental disorders as natural kinds is impeded by the theoryladenness of classification. See Cooper, Classifying Madness, ch. 3; R. Cooper, Psychiatry and Philosophy of Science (Montreal and Kingston: McGill-Queen's University Press, 2007), ch. 8. In this paper, I focus on her arguments concerning social and financial pressures.

${ }^{5}$ Cooper, Classifying Madness, 54.
} 
the way that the DSM has been shaped by the marketing of particular drug treatments. ${ }^{6}$ For instance, some mental disorders (e.g., panic disorder, social phobia) have been substantiated through marketing campaigns aimed to show that they can be successfully treated by particular drugs. Similarly, she suggests that niche marketing of certain mental disorders (e.g., attention deficit hyperactivity disorder) by pharmaceutical companies can function to raise the prominence (and prevalence) of a condition, thereby helping to establish its legitimacy as a mental disorder. According to Cooper these pressures from the pharmaceutical industry impede the DSM's aim of classifying natural kinds.

Cooper maintains that the medical insurance industry presents an even stronger impediment to classifying mental disorders as natural kinds. The negative influence of this industry on classification arises from the role that the DSM plays in medical insurance reimbursement. By the late 1960s, medical insurance carriers in the United States required a DSM diagnosis for patients to receive reimbursement for mental health treatment. ${ }^{7}$ According to Cooper, this created new pressures on the DSM, leading to new disorders (e.g., post-traumatic stress disorder) being included in DSM-III as the result of lobbying for certain groups to receive reimbursement for treatment. ${ }^{8}$ Moreover, insurance considerations have led to modifying diagnostic criteria for some disorders (e.g., seasonal affective disorder) in order to allow more patients to receive reimbursement for treatment. Cooper also suggests that pressures from the medical insurance industry can have more subtle effects on the DSM. For example, if psychiatrists routinely interpret certain diagnostic criteria less stringently in clinical practice in order to allow patients to receive reimbursement, then these shifts in clinical norms for what counts as a disorder could eventually be reflected back into the diagnostic criteria of the DSM.

\subsection{Murphy on Causal Classification}

Murphy's argument concerns the overarching approach to psychiatric classification adopted by the DSM. As will be discussed more extensively in the following section of this paper, since the publication of DSM-III in 1980, the DSM's classification system has been distinguished by its purely descriptive, operationalist, and atheoretical approach. Murphy argues that a more promising approach to psychiatric classification would be a causal and theoretical approach, which is informed by sciences such as biology and the cognitive neurosciences.

Against the descriptive approach to psychiatric classification currently favored by the DSM, Murphy charges that this system of classification is incoherent, heterogeneous, and provincial. Murphy argues that the descriptive approach is incoherent in its application of the medical model. While the DSM assumes that mental disorders are distinct (disease) entities that reflect underlying differences among individuals, it prohibits reference to the causal processes and dysfunctions that distinguish them. As Murphy puts it, "the assumption that disorders can be classified by symptomatology alone requires us to assume that a significant difference can exist

\footnotetext{
${ }^{6}$ Cooper articulates these arguments through a critical examination of the works of David Healy. See D. Healy The Antidepressant Era (Cambridge, MA: Harvard University Press, 1997); D. Healy, The Creation of

Psychopharmacology (Cambridge, MA: Harvard University Press, 2002).

${ }^{7}$ Cooper, Classifying Madness, 127-132.

${ }^{8}$ For an extensive discussion of the inclusion of post-traumatic stress disorder in DSM-III, see A. Young, The Harmony of Illusions: Inventing Post-Traumatic Stress Disorder (Princeton: Princeton University Press, 1995).
} 
between two individuals at the level of surface symptoms that does not reflect an underlying causal difference". ${ }^{9}$ With respect to heterogeneity, Murphy maintains that the DSM's symptombased approach to classification fails to distinguish heterogeneous conditions. This stems from the fact that DSM categories can group together different symptom profiles as manifestations of the same disorder (when there are multiple ways to satisfy the diagnostic criteria for a disorder), and the same symptoms can be produced by different underlying causes. Finally, Murphy complains that the DSM is provincial insofar as its concepts and terms (e.g., 'dissociation', 'selfesteem') are not coordinated with the sciences of the mind (e.g., the cognitive neurosciences). Without such engagement, the concepts and terms found in the DSM - which are often inherited from earlier historical traditions or folk psychology - are not subject to revision. Moreover, since mental disorders often involve dysfunctions that are studied extensively in the mind sciences, it is disadvantageous for the DSM to isolate itself from these bodies of research.

As a positive argument in favor of a causal approach to psychiatric classification, Murphy articulates an "exemplar" approach to classification, which is based on causal explanation. Murphy writes:

I argue for classification by exemplar, in which a disorder is classified according to both its symptoms and the explanation of those symptoms at a variety of levels of explanation .... Exemplars are representations of symptoms and course, so strictly speaking it is not exemplars alone but explained exemplars that should make up a nosology. . . . My approach classifies representations, and sorts people into classes based on their resemblance to the exemplars. ${ }^{10}$

The main difference between the DSM's approach to classification and Murphy's approach is that that the exemplar approach would supplement the descriptive categories of the DSM with causal information concerning the determinants of various symptoms. Exemplars include all the symptoms that arise from a particular (dysfunctional) causal process, which can help identify the proper boundaries of mental disorders. As Murphy puts it: "By articulating causal explanations . ..., the hope is that we will eventually be able [to] discriminate more finely among conditions that are currently lumped together by DSM's fuzzy categories, by distinguishing syndromes on the basis of etiology and pathology". ${ }^{11}$ In response to the objection that a causal system of taxonomy is difficult to achieve in practice due to lack of knowledge about etiology, Murphy emphasizes that a causal approach would point research in a fruitful direction. The immediate goal would be to identify disorders with distinct causes (causal discrimination), which would direct research towards finding out what those differences are; however, the long term goal would be to explain disorders by their distinct etiology (causal understanding). According to Murphy, even in the absence of mature causal theories, causal discrimination is an advance over clinical phenomenology insofar as it would orient research towards discovering what distinguishes different mental disorders (e.g., by developing specific tests, determining whether a disorder selectively responds to drug treatment). Ultimately, an exemplar approach could benefit clinical work insofar as clinicians could counter or prevent the normal outcome of pathological causal processes by using the causal histories associated with exemplars as a guide for intervention.

\footnotetext{
${ }^{9}$ Murphy, Psychiatry in the Scientific Image, 324.

${ }^{10}$ Murphy, Psychiatry in the Scientific Image, 345.

${ }^{11}$ Murphy, Psychiatry in the Scientific Image, 349-350.
} 


\section{The History of the DSM's Classification System}

A broader perspective for viewing Cooper's and Murphy's arguments can be achieved by considering relevant aspects of the history of the DSM. In the following section, I focus on psychiatry's "revolution in classification" associated with the publication of DSM-III, wherein the psychoanalytic and etiological system of classification characteristic of DSM-I and DSM-II was replaced with an "atheoretical" and descriptive system. Two significant historical factors responsible for this shift were the inadequacy of psychoanalytic classificatory concepts to meet emerging practical social needs, and the "neo-Kraepelinian" DSM-III task force led by Robert Spitzer, who aimed to promote the empirical, medical, and biological aspects of psychiatry.

\subsection{The DSM's Revolution in Classification}

DSM-I and DSM-II classified mental disorders in a broadly etiological manner, and they were theoretical manuals insofar as definitions and descriptions adopted in the manuals reflected key theoretical terms and distinctions of psychoanalytic psychiatry (most prominently, the psychoanalytic distinction between psychoses and neuroses).

DSM-I was published in 1952 and included 106 disorders. A fundamental etiological classificatory distinction of DSM-I is a division between: (1) mental disorders caused by brain impairment, and (2) mental disorders of psychogenic origin. ${ }^{12}$ In DSM-I, these two major groups of mental disorders are described as follows:

(1) those in which there is disturbance of mental function resulting from, or precipitated by, a primary impairment of the function of the brain, generally due to diffuse impairment of brain tissue; and

(2) those which are the result of a more general difficulty in adaptation of the individual, and in which any associated brain function disturbance is secondary to the psychiatric disorder. ${ }^{13}$

This division suggests a basic distinction between mental disorders with physical causes (i.e., brain damage) and mental disorders with psychological causes. In DSM-I, specific etiological factors are indicated in the name of all mental disorders caused by brain impairment (e.g., "acute brain syndrome due to metabolic disturbance", "chronic brain syndrome due to intracranial neoplasm", "chronic brain syndrome due to Mongolism"). By contrast, mental disorders of psychogenic origin (e.g., "psychotic disorders", "psychoneurotic disorders", "personality disorders") only include a brief description and offer no indication of possible etiology.

DSM-II was published in 1968 and included 182 disorders. Whereas DSM-I featured three major categories of mental disorders (see note 12), DSM-II organized mental disorders into

\footnotetext{
${ }^{12}$ The exact terminology of DSM-I is "disorders caused by or associated with impairment of brain tissue function" (APA, DSM-I, 2) and "disorders of psychogenic origin without clearly defined physical cause or structural change in the brain" (APA, DSM-I, 5). Besides these two major categories, DSM-I also features the third major category of "mental deficiency" (what would subsequently be called "mental retardation") of unknown cause.

${ }^{13}$ APA, DSM-I, 9.
} 
ten major categories. ${ }^{14}$ Despite this organizational expansion, DSM-II preserved the DSM-I distinction between disorders of brain impairment (referred to as "organic brain syndromes" in DSM-II) and disorders of psychogenic origin ("functional disorders"). ${ }^{15}$ The major categories of mental disorders that indicate specific etiological factors in DSM-II are mental retardation (e.g., "mental retardation caused by chromosomal abnormality") and organic brain syndromes (e.g., "psychosis associated with intracranial infection"). For the remaining eight major categories of mental disorders (see note 14), DSM-II only offers brief descriptions of the disorders without mentioning any specific causes, although these functional disorders are generally understood as disorders with psychological causes.

The publication of DSM-III marked a revolution in psychiatric classification due to its rejection of the theoretical (i.e., psychoanalytic) and etiological approach of its predecessors, and adoption of an atheoretical and descriptive approach. In the introduction of DSM-III, it is announced that:

The approach taken in DSM-III is atheoretical with regard to etiology ... except for those disorders for which this is well established ... The major justification for the generally atheoretical approach ... is that the inclusion of etiological theories would be an obstacle to use of the manual by clinicians of varying theoretical orientations ... Because DSM-III is generally atheoretical with regard to etiology, it attempts to describe comprehensively what the manifestations of the mental disorders are, and only rarely attempts to account for how the disturbances come about ... This approach can be said to be "descriptive" in that the definitions of the disorders generally consist of descriptions of the clinical features of the disorders. ... at the lowest order of inference necessary to describe the characteristic features of the disorder. ${ }^{16}$

The most significant innovation of DSM-III was the introduction of the now familiar "diagnostic criteria", in which a diagnosis for a mental disorder can only be made if a set of necessary and sufficient criteria (typically observable or reportable symptoms) are satisfied. In addition to the introduction of diagnostic criteria in DSM-III, the major categories of mental disorder expanded to fifteen, while the total number of mental disorders listed increased to 265. Another prominent difference in DSM-III is that much of the psychoanalytic terminology of DSM-I and DSM-II was replaced with theoretically neutral language. While DSM-III does indicate etiological factors for a handful of disorders (mainly among the category of "organic mental disorders"), the key etiological distinction between disorders caused by brain impairment and disorders of psychogenic origin (or "functional disorders") was removed. Thus, for the most part, DSM-III followed its promise of providing an atheoretical and descriptive approach to classification, which has remained the status quo in subsequent editions.

\footnotetext{
${ }^{14}$ APA, DSM-II. The major categories in DSM-II are: (1) mental retardation, (2) organic brain syndromes, (3) psychoses not attributed to physical conditions, (4) neuroses, (5) personality disorders, (6) psychophysiologic disorders, (7) special symptoms, (8) transient situational disturbances, (9) behavioral disorders of childhood and adolescence, and (10) conditions without manifest psychiatric disorder and non-specific conditions.

${ }^{15}$ See APA, DSM-II, 123. For a summary of how DSM-I classifications map onto DSM-II classifications, see APA, DSM-II, 65-82, 125.

${ }^{16}$ APA, DSM-III, 7, emphasis in original.
} 


\subsection{The Marginalization of Psychoanalysis from 1960-1980}

The most important historical factors responsible for the DSM's revolution in classification are related to the decline of psychoanalytically-oriented psychiatry in the United States, especially in the 1960s and 1970s. ${ }^{17}$ While psychoanalysis enjoyed a period of prestige in American from the post-war period to the early $1960 \mathrm{~s},{ }^{18}$ after the 1960 s, its scientific legitimacy was increasingly called into question. The 1960s featured prominent criticisms of psychiatry by "antipsychiatrists" such as Thomas Szasz and R. D. Laing, ${ }^{19}$ who questioned the scientific adequacy of concepts such as mental illness and mental health, as well as Michel Foucault, who suggested that the allegedly more humane medical conception of madness associated with modern psychiatry was merely another form of "moral confinement" ${ }^{20}$ Psychiatry was also in the middle of several public embarrassments in the 1970s. In DSM-II, homosexuality was classified as a mental disorder, ${ }^{21}$ and gay rights organizations lobbied to have this classification removed. In 1973, the APA boards of trustees decided - on apparently political, rather than scientific grounds - to remove the classification. ${ }^{22}$ It was also in 1973 when David Rosenhan published a highly publicized study, ${ }^{23}$ in which confederates feigning mental illness gained admission to mental hospitals across the United States, and were not recognized by hospital staff as normal, in spite of the fact that they acted normally after the initial interview. Moreover, psychoanalyticallyoriented psychiatry faced criticism from within psychiatry by disenchanted biologically-oriented psychiatrists who wished to narrow the scope of the field and raise psychiatry to the standards of acceptable science and medicine. ${ }^{24}$

The status of psychoanalytic psychiatry in the 1960s and 1970s was further marginalized by its inability to meet certain practical societal needs. In the two decades following 1960, there was a dramatic drop in the number of institutionalized psychiatric patients in state-run mental hospitals across the United States. ${ }^{25}$ A key reason for this widespread deinstitutionalization of patients was the introduction of chlorpromazine, an anti-psychotic drug used to treat psychosis (one of the main symptoms of schizophrenia). After the introduction of chlorpromazine in the

\footnotetext{
${ }^{17}$ In this paper, I use the term "psychoanalysis" to refer to the psychodynamic and psychosocial psychiatric paradigm - associated with a historical lineage from Sigmund Freud to Adolf Meyer - that was dominant from 1945 to 1975. For a more extensive discussion of this paradigm, see G. N. Grob, "Origins of DSM-I: A Study in Appearance and Reality”, American Journal of Psychiatry 148 (1991), 421—431; M. Wilson, "DSM-III and the Transformation of American Psychiatry: A History”, American Journal of Psychiatry 150 (1993), 399-410.

${ }^{18}$ See N. G. Hale, The Rise and Crisis of Psychoanalysis in America: Freud and the Americans, 1917-1985 (Oxford: Oxford University Press, 1995); Grob, "Origins of DSM-I".

${ }^{19}$ T. S. Szasz, The Myth of Mental Illness: Foundations of a Theory of Personal Conduct (New York: Paul B. Hoeber, 1961); R. D. Laing, The Divided Self: An Existential Study in Sanity and Madness (London: Tavistock Publications, 1959).

${ }^{20}$ M. Foucault, Folie et Déraison: Histoire de la folie a l'âge classique (Paris: Plon, 1961). Foucault’s work was introduced to the United States by the significantly abridged English translation, Madness and Civilization: A History of Insanity in the Age of Reason, trans. R. Howard (New York: Pantheon, 1965).

${ }^{21}$ APA, DSM-II, 44.

${ }^{22}$ See R. Bayer, Homosexuality and American Psychiatry: The Politics of Diagnosis (New York: Basic Books, 1981); R. Bayer and R. L. Spitzer, "Edited Correspondence on the Status of Homosexuality in DSM-III”, Journal of the History of the Behavioral Sciences 18 (1982), 32-52.

${ }^{23}$ D. Rosenhan, “On Being Sane in Insane Places”, Science 179 (1973), 250-258.

${ }^{24}$ See Wilson, "DSM-III and the Transformation"; R. Mayes and A. V. Horwitz, "DSM-III and the Revolution in the Classification of Mental Illness", Journal of the History of the Behavioral Sciences 41 (2005), 249-267.

${ }^{25}$ See G. N. Grob, From Asylum to Community: Mental Health Policy in Modern America (Princeton, NJ: Princeton University Press, 1991); G. N. Grob, “The Paradox of Deinstitutionalization”, Society 32 (1995), 51—59.
} 
1950s, many institutionalized patients no longer needed to be confined to asylums, and were able to live in community settings. The increased deinstitutionalization and use of drugs to treat mental disorders (viz., psychosis, depression, anxiety, manic-depressive disorder) in the 1960s and 1970s highlighted the psychoanalytic paradigm's inability to treat more serious psychiatric disorders. ${ }^{26}$ In the 1970 s, psychoanalysis was also facing criticism from third-party medical insurance companies, who demanded clarity in psychiatric diagnoses and accountability for treatment outcomes. ${ }^{27}$ By the mid-1970s, insurance companies such as Blue-Cross and Aetna cut financial support for psychotherapy significantly, citing lack of clarity and uniformity of diagnoses for mental disorders, as well as lack of clinical accountability in the mental health care system. ${ }^{28}$ As such, the psychoanalytic diagnostic categories of DSM-II were failing to meet third-party insurance companies’ need for clear and standardized psychiatric diagnoses.

In his well-known study of scientific revolutions, Thomas Kuhn suggested that conceptual revolutions are typically preceded by a stage of crisis, in which a paradigm's inability to solve certain problems are recognized in the scientific community. ${ }^{29}$ This was certainly the case in the DSM's revolution in classification, in which the inadequacies of psychoanalysis from both an epistemic and pragmatic view - were recognized from both inside and outside of psychiatry by the mid-1970s. It was in this historical context that the APA task force responsible for revising DSM-II was able to transform the DSM from a psychoanalytic and etiological system of classification to an atheoretical and descriptive system.

\subsection{DSM-III: The Neo-Kraepelinian Response to Psychiatry’s Crisis}

In 1974, Robert L. Spitzer was appointed as the chairman for the APA Task Force on Nomenclature and Statistics, which was officially formed to coordinate DSM-III with the ninth edition of the World Health Organization's International Classification of Diseases (ICD), ${ }^{30}$ and update the manual to reflect the current state of knowledge on mental disorders. ${ }^{31}$ In less than a decade, Spitzer and his like-minded task force reinvented the DSM in accordance with their “neo-Kraepelinian” outlook on psychiatric classification. ${ }^{32}$ The "neo-Kraepelinian” label was originally coined by George Klerman, who reported a Kraepelinian revival in psychiatry (led by

\footnotetext{
${ }^{26}$ Mayes and Horwitz, "DSM-III and the Revolution”, 254-255.

${ }^{27}$ A. V. Horwitz, Creating Mental Illness (Chicago: University of Chicago Press, 2002), ch. 3.

${ }^{28}$ Wilson, "DSM-III and the Transformation", 403.

${ }^{29}$ T. S. Kuhn, The Structure of Scientific Revolutions, $3^{\text {rd }}$ ed. (Chicago: University of Chicago Press, 1996), chs. 78. For discussion of American psychiatry's revolution in classification in Kuhnian terms, see G. L. Klerman, "Paradigm Shifts in USA Psychiatric Epidemiology Since World War II”, Social Psychiatry 25 (1990), 27-32; Horwitz, Creating Mental Illness, ch. 3.

${ }^{30}$ WHO, Manual of the International Statistical Classification of Diseases, Injuries and Causes of Death (Geneva: World Health Organization, 1977). In this paper, I refer to this manual as ICD-9.

${ }^{31}$ APA, DSM-III, $1-2$.

32 Roger K. Blashfield, The Classification of Psychopathology: Neo-Kraepelinian and Quantitative Approaches (New York: Plenum Press, 1984) argues that Spitzer's appointed task force formed an "invisible college" insofar as the group had close professional and social allegiances with one another, although these ties were not obvious to outsiders. Spitzer had especially close ties to a research group at Washington University in St. Louis who developed the influential "Feighner criteria", a set of diagnostic criteria for fifteen mental disorders, which effectively served as a prototype for DSM-III diagnoses. See J. P. Feighner, E. Robins, S. B. Guze, R. A. Woodruff, G. Winokur, and R. Munoz, "Diagnostic Criteria for Use in Psychiatric Research”, Archives of General Psychiatry 26 (1972), 57_63.
} 
Samuel Guze, Eli Robins, George Winokur, Donald Klein, and Robert Spitzer) accompanying the decline of Meyerian and Freudian psychoanalysis. ${ }^{33}$ This movement continued the tradition of psychiatry associated with Emil Kraepelin: the German psychiatrist who developed a biological approach to psychiatry in the late-nineteenth and early-twentieth century that was opposed to Freudian psychoanalysis. ${ }^{34}$ In the psychoanalytic paradigm, it was assumed that psychological mechanisms play a crucial role in the emergence of mental illness, and the boundary between normality and abnormality is continuous (ranging from neuroses of mild severity to psychoses of major severity). ${ }^{35}$ By contrast, the neo-Kraepelinians assumed that mental illnesses are discrete physical (biological) diseases, and there is a clear boundary between normality and the sick. ${ }^{36}$ Within this framework, it was crucial to distinguish the mentally ill from the well with reliable and valid diagnostic categories, which in psychoanalysis was secondary to the goal of elucidating the meaning of individuals' observed symptoms. ${ }^{37}$ The desideratum for empirically validated diagnostic categories motivated the neo-Kraepelinian belief that mental disorders should be classified on the basis of their observable symptoms, as opposed to speculative inferences regarding their causes. ${ }^{38}$ These neo-Kraepelinian tenets formed the foundation for the task force's shared conviction in the importance of descriptive diagnostic classifications.

The neo-Kraepelinian principles that guided the APA task force played a central role in influencing how the DSM would be revised, and the agenda of this task force clearly went beyond coordinating DSM-III with ICD-9. An excerpt from the minutes of the first meeting of the task force in 1974 (recorded by Spitzer) indicates that a revolution was already in the making:

It was the unanimous opinion of the Committee that etiology should be a classificatory principle only when it is clearly known ... The diagnostic manual will be essentially behavioral, with exception for conditions of known etiology . . . . It was agreed that "functional" is no longer a suitable designation for a group of conditions - schizophrenias and affective disorders - which are no longer seen as purely psychogenic. It was further agreed that "psychosis" and "neurosis" are useful possibly as adjectives, but not as classificatory principles. ${ }^{39}$

In 1975, after a preliminary version of DSM-III was drafted, which included significantly more diagnoses and more detailed descriptions of disorders than DSM-II, objections were raised by

\footnotetext{
${ }^{33}$ G. L. Klerman, “The Evolution of Scientific Nosology” in J. C. Shershow (ed.), Schizophrenia: Science and Practice (Cambridge, MA: Harvard University Press, 1978), 99-121. Spitzer, "Letter to the Editor”, Schizophrenia Bulletin 8 (1982), 592, has insisted that he is not a "neo-Kraepelinian", although he admits that his task force was made up of (self-identifying) neo-Kraepelinians. For discussion of how closely the APA task force followed Kraepelin’s principles, see H. S. Decker, “How Kraepelinian was Kraepelin? How Kraepelinian are the neo-Kraepelinians? - from Emil Kraepelin to DSM-III”, History of Psychiatry 18 (2007), 337-360.

${ }^{34}$ See Blashfield, Classification of Psychopathology, ch. 1.

${ }^{35}$ Wilson, "DSM-III and the Transformation”, 400-401.

${ }^{36}$ See Klerman, “The Evolution of Scientific Nosology”, 104-105.

${ }^{37}$ Wilson, “DSM-III and the Transformation”, 402.

38 See Mayes and Horwitz, “DSM-III and the Revolution”, 259-261.

${ }^{39}$ Cited in Wilson, "DSM-III and the Transformation”, 405.
} 
clinically-oriented (including psychoanalytic) psychiatrists who feared that DSM-III would be useful to researchers, but useless to clinicians. ${ }^{40}$ This led to the formation of a liaison committee (made up of clinically-oriented psychiatrists), and culminated in a controversy in 1979 centered on the task force's attempted removal of the term "neurosis" as a classificatory principle. ${ }^{41}$ At this point, DSM-III was in its final stages and in danger of not being approved by the APA unless psychiatrists who wanted "neurosis" to be reinstated in the manual could be appeased. ${ }^{42}$ This conflict stemmed from the fact that "[p]sychoanalytic practitioners feared that a change in psychiatric nomenclature might result in a challenge by third-party reimbursement sources seeking to limit payment to patients receiving long-term therapy". ${ }^{3}$ The task force eventually compromised (and was able to publish DSM-III) by retaining the term "neurosis", but qualifying that the term was only being used in a descriptive manner (rather than referring to an etiological process). ${ }^{44}$

DSM-III was largely successful in responding to the difficulties that psychoanalytic psychiatry faced in the 1960s and 1970s. Against accusations of pseudo-science, the neoKraepelinian agenda of reorienting the DSM away from psychoanalysis and towards a biologically-oriented psychiatry alleviated some of these epistemic concerns by reaffirming the empirical, medical, and biological aspects of psychiatry. ${ }^{45}$ On a more specific level, DSM-III's promise of reliable and valid psychiatric diagnoses, along with the assumption that mental disorders were a subset of physical illnesses (see note 57), was consonant with the emerging demands of prescribing pharmaceutical drugs, ${ }^{46}$ and met the medical insurance industry’s need to reimburse individuals who had genuine medical problems. With respect to the latter, during the DSM revision process, representatives from Blue Cross and Aetna were in contact with Spitzer's task force, and encouraged them to standardize the manual's diagnostic criteria so insurers could distinguish medical conditions from non-psychiatric problems. ${ }^{47}$ Several members of the task force have acknowledged that DSM-III's structure was "strongly influenced by the need for diagnoses for which insurance companies could provide reimbursement”. ${ }^{4}$

\footnotetext{
${ }^{40}$ Wilson, "DSM-III and the Transformation”, 405-407.

${ }^{41}$ See Mayes and Horwitz, “DSM-III and the Revolution”, 261-263.

${ }^{42}$ R. Bayer and R. L. Spitzer, "Neurosis, Psychodynamics, and DSM-III: A History of the Controversy", Archives of General Psychiatry 42 (1985), 187-198.

${ }^{43}$ Bayer and Spitzer, "Neurosis, Psychodynamics, and DSM-III”, 192.

${ }^{44}$ APA, DSM-III, 9-10.

${ }^{45}$ Wilson, "DSM-III and the Transformation”, 408-409.

${ }^{46}$ Cooper, Classifying Madness, 112-118, argues that there is little historical evidence to support the idea that DSM-III was intentionally fashioned to facilitate research in pharmacology. However, the standardized diagnostic categories of DSM-III were undoubtedly regarded as useful tools for pharmaceutical treatment practices insofar as they could reliably diagnose more serious disorders such as schizophrenia and bipolar disorder.

${ }^{47}$ S. S. Sharfstein, “Third-Party Payments, Cost Containment, and DSM-III” in G. L. Tischler (ed.), Diagnosis and Classification in Psychiatry: A Critical Appraisal of DSM-III (Cambridge: Cambridge University Press, 1987), 530 - 538. Cooper, Classifying Madness, 132 — 134, suggests that although DSM-III was clearly shaped by the medical insurance industry, the APA and Spitzer's task force denied this influence in public venues in order to uphold the scientific purity of DSM-III and, more generally, psychiatry.

${ }^{48}$ Cited in Mayes and Horwitz, “DSM-III and the Revolution”, 262.
} 


\section{Lessons from the History of Psychiatry for Philosophers}

The history that surrounds the publication of DSM-III highlights the fact that psychiatry is an applied science that is focused on addressing a number of practical problems. Besides addressing purely epistemic problems concerning how to classify mental disorders, the DSM is a manual that simultaneously needed to address concrete practical problems (e.g., providing a manual that could facilitate mental health treatment). In the following section, I argue that evaluating a system of psychiatric classification should consider a system's capacity to address practical problems (e.g., facilitating treatment goals) as well as epistemic ones (e.g., individuating mental disorders that are natural kinds), which is an issue that is often overlooked in philosophical analyses. From this perspective, I critically assess and expand upon the analyses advanced by Cooper and Murphy. I suggest that a consideration of the history of the DSM brings into question Cooper's privileging of epistemic over practical values, and can reinforce Murphy's advocacy of a causal system of psychiatric classification.

\subsection{The Pragmatic Aspects of Psychiatric Classification}

Psychiatry's revolution in classification is a useful case study for indicating some ways in which Thomas Kuhn's theory of scientific revolutions can be elaborated. In his analysis, Kuhn emphasized how successor paradigms are more effective at solving puzzles than prior paradigms. ${ }^{49}$ In responding to charges of relativism, Kuhn countered that: "[l]ater scientific theories are better than earlier ones for solving puzzles in often quite different environments.... That is not a relativist's position, and it displays the sense in which I am a convinced believer in scientific progress" ${ }^{50}$ Here, Kuhn presents "puzzle-solving efficacy" as a defensible criterion for judging “scientific progress”. For my purposes, I want to highlight the fact that Kuhn's discussion of puzzle-solving is made in purely epistemic terms. In discussing the standards that are used to judge between competing paradigms, Kuhn consistently cited a number of values: (1) empirical accuracy, (2) internal and external consistency, (3) explanatory scope, (4) simplicity, and (5) fruitfulness for research. ${ }^{51}$ These values cited by Kuhn are purely epistemic insofar as they are assumed to promote good knowledge claims; however, Kuhn acknowledged that his list was not exhaustive and that "[o]ther sorts of values exist as well - for example, science should (or need not) be socially useful". ${ }^{52}$ As illustrated by the DSM's revolution in classification, nonepistemic values (e.g., being useful for the medical insurance industry) can play a central role in determining which paradigms emerge as victors. This suggests that Kuhn's notion of puzzlesolving need not be limited to epistemic problems, and can extend to practical problems.

Besides being a historical factor in determining which paradigms survive, I want to suggest that in some fields of science - especially in applied fields of science, such as psychiatry - the capacity of a paradigm to solve practical problems is an important value to consider in

\footnotetext{
${ }^{49}$ Kuhn, Structure of Scientific Revolutions, ch. 13.

${ }^{50}$ Kuhn, Structure of Scientific Revolutions, 206.

${ }^{51}$ Kuhn, Structure of Scientific Revolutions, 184-185; T. S. Kuhn, "Objectivity, Value Judgment, and Theory Choice" in T. S. Kuhn, The Essential Tension: Selected Studies in Scientific Tradition and Change (Chicago: University of Chicago Press, 1977), 320-329; T. S. Kuhn, "Rationality and Theory Choice”, Journal of Philosophy 80 (1983), 563-570.

${ }^{52}$ Kuhn, Structure of Scientific Revolutions, 185, emphasis added.
} 
evaluating the relative merits of competing theoretical frameworks. ${ }^{53}$ Given that the benefits of applied sciences (e.g., medicine, engineering) arise primarily from the success of their concrete applications, it is reasonable to evaluate the relative merits of competing theoretical systems in these domains in terms of the ability of a paradigm to solve practical problems. ${ }^{54}$ From this perspective, the historical fact that practical considerations played a crucial role in the evolution of the DSM provides support for the philosophical argument that theoretical frameworks in the applied sciences should be evaluated on pragmatic grounds. ${ }^{55}$

In this connection, I submit that the neo-Kraepelinian classification system of DSM-III was superior to the psychoanalytic and etiological system it replaced insofar as it was a more useful manual for addressing practical problems. In particular, a key practical problem that the psychoanalytic paradigm was unable to solve, and the neo-Kraepelinian paradigm was able solve, was the provision of a system of classification that could accommodate the needs of the medical insurance industry (i.e., by providing clear and reliable diagnoses). Furthermore, I would suggest that DSM-III implicitly assumed a value-orientation that weighed practical goals more heavily than DSM-I and DSM-II insofar as the authors of DSM-III attempted to provide a diagnostic manual that could be used by researchers of different theoretical orientations, encourage the study of mental disorders in a standardized fashion, and provide reliable diagnoses for purposes of treatment.

\subsection{Classifying Natural Kinds vs. Promoting Practical Goals}

Cooper argues that the DSM's goal of classifying mental disorders as natural kinds is unlikely to be realized because of external influences on the manual (e.g., the medical insurance industry). While Cooper reaches this conclusion through a close engagement with the history surrounding the publication of DSM-III, ${ }^{56}$ and her discussion of specific undesirable influences on the DSM is unobjectionable, her analysis fails to sufficiently acknowledge the inherently practical elements of psychiatric classification. What the history of the DSM illustrates is the importance of non-epistemic goals in psychiatry. In what follows, I argue that Cooper's philosophical analysis places too much weight on epistemic goals at the expense of practical goals in its

\footnotetext{
${ }^{53}$ I assume that pure science concerns describing how the world is, whereas applied science concerns changing the world. This understanding of the pure and applied science distinction is defended by M. Bunge, "Technology as Applied Science”, Technology and Culture 7 (1966), 329-349. Philosophers of science who have emphasized the importance of practical and social dimensions of scientific knowledge include Helen Longino and Ronald Giere. See H. E. Longino, The Fate of Knowledge (Princeton, NJ: Princeton University Press, 2002); R. N. Giere, Scientific Perspectivism (Chicago: University of Chicago Press, 2006).

${ }^{54}$ For a more comprehensive discussion of this issue with reference to specific instances of applied science, see K. Shrader-Frechette, "Hydrogeology and Framing Questions Having Policy Consequences", Philosophy of Science 64 (1997), S149—S160; M. Carrier, "Knowledge and Control: On the Bearing of Epistemic Values in Applied Science” in P. Machamer and G. Wolters (eds.), Science, Values, and Objectivity (Pittsburgh, PA: University of Pittsburgh Press, 2004), 275-293; I. de Melo Martín and K. Intemann, "Can Ethical Reasoning Contribute to Better Epidemiology? A Case Study in Research on Racial Health Disparities”, European Journal of Epidemiology 22 (2007), 215-221.

${ }^{55}$ This stance follows the Kuhnnian view that descriptive generalizations from the history of science can sometimes serve as evidence for philosophical prescriptions concerning what constitutes good scientific practice. See T. S. Kuhn, "Reflection on My Critics" in I. Lakatos and A. Musgrave (eds.), Criticism and the Growth of Knowledge (Cambridge: Cambridge University Press, 1970), 237; Kuhn, Structure of Scientific Revolutions, 207-208.

${ }^{56}$ Cooper, Classifying Madness, 127-138.
} 
implicit assumptions that: (1) the primary aim of the DSM is to classify natural kinds, and (2) it is always undesirable for this aim to be constrained by non-epistemic factors.

While there is some credibility to Cooper's assumption that the main goal of the DSM is to classify natural kinds, her analysis obscures other goals of the manual. The authors of the DSM have never discussed mental disorders in the language of "natural kinds"; however, a guiding assumption of the DSM-III task force was the Kraepelian assumption that "mental disorders are a subset of medical conditions". 57 This indicates that an implicit aim of DSM-III was to classify mental disorders that are naturally occurring medical conditions (i.e., natural kinds), as opposed to various forms of deviance that might have a social or cultural basis (i.e., artificial kinds). Although classifying natural kinds is an implicit goal of the DSM, it is clear that it aims to achieve other goals. One of the clearest and most explicit statements of the DSM's goals is stated in DSM-IV-TR as follows: "The purpose of DSM-IV is to provide clear descriptions of diagnostic categories in order to enable clinicians and investigators to diagnose, communicate about, study, and treat people with various mental disorders". ${ }^{8}$ In this passage, and the section discussing DSM-IV's definition of mental disorder, ${ }^{59}$ there is no indication that the DSM primarily aims to classify naturally occurring disorders. ${ }^{60}$ It is also notable that facilitating the treatment of individuals with mental disorders, which is a practical and nonepistemic goal, is explicitly cited by the authors of DSM-IV as a goal.

Questions concerning the actual aims of the DSM may be a red-herring given that - as Cooper correctly notes in her analysis - the DSM's public image does not always square with its authors' intentions. ${ }^{61}$ However, I want to more decisively challenge Cooper's implicit assumption that the DSM ought to classify natural kinds, and that non-epistemic influences that detract from this goal are necessarily undesirable. As the history of the DSM-III revision process illustrates, considerations involved in revising the DSM's system of classification (e.g., meeting the emerging demands of the medical insurance industry) were not limited to creating a coherent epistemic taxonomic system. Cooper regards the influence of these non-epistemic forces as negative, but is this necessarily the case? To take one of Cooper's examples, suppose that pharmaceutical industry creates pressures to lump together disorders (e.g., as "affective spectrum disorders") that respond to similar treatments. ${ }^{62}$ While this phenomenon may detract from the

\footnotetext{
${ }^{57}$ See R. L. Spitzer, M. Sheehy and J. Endicott, “DSM-III: Guiding Principles” in V. M. Rakoff, H. C. Stancer, and H. B. Kedward (eds.), Psychiatric Diagnosis (New York: Brunner/ Mazel, 1977), 1-24; R. L. Spitzer and J. Endicott, "Medical and Mental Disorder: Proposed Definition and Criteria” in R. L. Spitzer and D. F. Klein (eds.), Critical Issues in Psychiatric Diagnosis (New York: Raven Press, 1978), 15—39. The APA task force initially planned to include the statement that "mental disorders are a subset of medical conditions" in the introduction of DSM-III; however, this plan was voted against since psychologists, social workers, and counselors regarded it as a declaration that psychiatrists (with medical training) were solely responsible for the treatment of mental disorders. See Mayes and Horwitz, "DSM-III and the Revolution”, 260-261.

${ }^{58}$ APA, DSM-IV-TR, xxxvii, emphasis added.

${ }^{59}$ APA, DSM-IV-TR, xxx- $\mathrm{xxxi}$.

${ }^{60}$ Moreover, in discussing limitations of the categorical approach, the authors of DSM-IV-TR, xxxi, explicitly eschew the idea that they are classifying natural kinds in Cooper's sense:

In DSM-IV, there is no assumption that each category of mental disorder is a completely discrete entity with absolute boundaries dividing it from other mental disorders or from no mental disorder. There is also no assumption that all individuals described as having the same mental disorder are alike in all important ways.

${ }^{61}$ Cooper, Classifying Madness, 132-133.

${ }^{62}$ Cooper, Classifying Madness, 124-125.
} 
goal of distinguishing natural kinds, it may promote the treatment of individuals (i.e., by distinguishing disorders on the basis of successful interventions). As indicated in DSM-IV, one goal of the DSM - besides promoting epistemic research goals - is to facilitate treatment goals. I believe that this is a perfectly defensible goal for evaluating the DSM. As argued above, there are compelling reasons for evaluating theoretical systems in applied sciences, such as systems of psychiatric classification, on practical grounds (e.g., the capacity of a system to facilitate treatment goals). Hence, I reject Cooper’s implicit assumption that epistemic goals (i.e., classifying mental disorders as natural kinds) should be weighted more heavily than practical goals (e.g., providing a manual that facilitates treatment and clinical intervention). The history of the DSM demonstrates that non-epistemic influences are often ubiquitous and inevitable, as opposed to factors that can (or need to) be removed or minimized.

\subsection{The Historicity of Psychiatric Classification}

The historical and practical aspects of psychiatric classification emphasized in this paper can serve to strengthen Murphy's general advocacy of a causal system of classification. One insight that can be gained from looking at the history of the DSM is that the move to a descriptive system of classification in DSM-III was largely due to contingent historical factors (viz., the theoretical inadequacy of DSM-I and DSM-II, and the inability of DSM-II to meet certain social needs) specific to the 1960s and 1970s. This suggests that there is no absolute and ahistorical ideal of psychiatric classification that will be appropriate for all historical periods. While the descriptive system of classification introduced in DSM-III may have been an improvement over DSM-II, it does not need to be regarded as an end-point.

The historical nature of psychiatric taxonomic systems was a fact that was fully appreciated by the philosopher of science, Carl Hempel. ${ }^{63}$ According to Hempel, the development of a taxonomic system is a historical process, which must begin with a descriptive stage before progressing to a theoretical stage. He writes:

[T]he development of a scientific discipline may often be said to proceed from an initial ... stage, which primarily seeks to describe the phenomena under study . . . to subsequent more and more 'theoretical' stages, in which increasing emphasis is placed upon the attainment of comprehensive theoretical accounts. ... In medical science, the development from a predominantly descriptive to an increasingly theoretical emphasis is reflected ... in the transition from a largely symptomatological to a more and more etiological point of view. ${ }^{64}$

In its descriptive stage, Hempel recommends the use of empirically-defined operational concepts, and he remarks that the psychoanalytic classificatory concepts of DSM-I are problematic insofar as they "lack clear and uniform criteria of application". ${ }^{65}$ While Hempel

\footnotetext{
${ }^{63}$ C. G. Hempel, "Fundamentals of Taxonomy" in C. G. Hempel, Aspects of Scientific Explanation and Other Essays in the Philosophy of Science (New York: The Free Press, 1965), 137-154. Hempel's analysis is particularly interesting as it was originally written in 1959, located historically between the publications of DSM-I (1952) and DSM-II (1968), and prior to the DSM’s descriptive turn.

${ }^{64}$ Hempel, "Fundamentals of Taxonomy", 140.

${ }^{65}$ Hempel, "Fundamentals of Taxonomy", 141.
} 
would have approved of the diagnostic criteria introduced in DSM-III, his analysis suggests that to achieve a truly scientific status, a system of classification cannot remain content in its descriptive stages, but must advance to theoretical stages. For Hempel, mature taxonomic systems should organize subclasses by theoretical principles, which provide a basis for explanation and prediction, ${ }^{66}$ and he cites examples from chemistry and biology in support of this stance ${ }^{67}$ From this Hempelian standpoint, a theoretical and causal system of classification such as Murphy's exemplar approach would be the next logical stage to supplant the descriptive system of classification currently employed in the DSM. ${ }^{68}$

The history of psychiatry illustrates that the DSM's descriptive system of classification was appropriate in the historical context of the late-1970s, when the psychoanalytic diagnostic categories of DSM-II were failing to meet the emerging need for clear and reliable diagnostic categories. In the context of current mental health practices, however, the historical factors that motivated the atheoretical and descriptive approach (i.e., removing questionable psychoanalytic assumptions, avoiding excessive speculation about etiology, providing reliable diagnostic categories) are no longer relevant. From this standpoint, there are no longer strong reasons for not supplementing the descriptive diagnostic categories of the DSM with causal information. As Murphy points out, the prohibition against theoretical and causal information has functioned - in contemporary practices - to isolate the DSM from large bodies of scientific research on psychopathology, which could provide crucial theoretical resources for discriminating distinct disorders (i.e., by etiology). Moreover, a theoretical and causal basis for psychiatric classification - in its capacity to provide explanatory and predictive classificatory concepts - could provide a more robust basis for testing and revising diagnostic categories in the DSM.

Like Cooper’s analysis, Murphy’s argument tends to emphasize epistemic goals, and downplay the importance of practical goals. However, the practical aspects of the DSM can also support Murphy's argument for a causal system of classification. As mentioned previously, the DSM explicitly cites the facilitation of treatment as an important pragmatic function of the DSM. ${ }^{69}$ In this connection, causal information about mental disorders could provide useful guidance for successfully treating individuals with mental disorders. A causal system could incorporate treatment goals by grouping together disorders that involve similar causal factors (e.g., similar neurotransmitter dysfunction) or disorders that respond positively to similar treatments (e.g., cognitive-behavioral therapy). ${ }^{70}$ With respect to the argument that "inclusion of etiological theories would be an obstacle to use of the manual by clinicians of varying theoretical

\footnotetext{
${ }^{66}$ Hempel, “Fundamentals of Taxonomy”, 146-151. In this connection, Hempel, praises DSM-I for its adoption of an "etiological or generally theoretical account", 149.

${ }^{67}$ Hempel, "Fundamentals of Taxonomy", 147-148. Hempel remarks that the modern periodic table of elements is organized by theoretical principles such that the subclasses of the table (e.g., the vertical columns or "groups") reflect properties in the atomic structure of the elements. He also points out that while early biological taxonomic systems were purely descriptive insofar as its subclasses were determined exclusively by morphological characteristics - with the advent of the theory of evolution - this morphological basis was replaced by a classification system with a stronger theoretical (i.e., phylogenetic) basis.

${ }^{68}$ Murphy, Psychiatry in the Scientific Image, 220-221, recognizes the relevance and importance of Hempel's essay for his own argument for causal classification.

${ }^{69}$ APA, DSM-IV-TR, xxxvii.

${ }^{70}$ Hempel, "Fundamentals of Taxonomy”, 149—150, discusses the possibility of including prognostic prospects and therapeutic possibilities among the defining characteristics of mental disorders.
} 
orientations”, ${ }^{71}$ I agree with Murphy's contention that a move towards causal classification would still represent an advance over purely descriptive classifications insofar as it would provide a systematic basis for revising diagnostic categories and suggesting fruitful lines of research. At present, the DSM's descriptive system provides no clear guidelines for deciding when a disorder should be retained, revised, or removed from the manual. By contrast, in a causal system such as Murphy's exemplar approach, possession of a distinctive biological etiology (even if that etiology is currently unknown) could serve as a relatively clear heuristic for determining when a disorder should be included or not. With respect to the theoretical basis for such a system, I would suggest that the only tenable theoretical basis for a causal system of classification would be a pluralistic one, constituted by the best current theories (e.g., in neuroscience, molecular biology, the behavioral sciences), and always subject to revision.

The argument for a causal approach to psychiatric classification is particularly relevant for proposed changes in DSM-V (which is scheduled to be published in 2013). Members of the DSM-V task force (chaired by David J. Kupfer) have explicitly stated their intention to move the classification system of the DSM away from the descriptive "neo-Kraepelinian diagnostic paradigm" adopted in DSM-III towards a more theoretical approach. ${ }^{72}$ One of the explicit motivations for such a shift - consonant with the arguments above concerning the fallibility and revisability of diagnostic categories - is to provide a system of classification that would "facilitate ongoing testing of the diagnostic criteria that are intended to be scientific hypotheses". ${ }^{73}$ While DSM-V will not include causal information about mental disorders (the main difference between DSM-IV and DSM-V will be more prominent use of dimensional measures), members of the DSM-V task force have anticipated such a possibility. ${ }^{74}$ The analysis of this paper supports a movement towards a more theoretical and causal approach to psychiatric classification.

\section{Conclusion}

In this paper, I critically examined Cooper's and Murphy's analyses of psychiatric classification by considering relevant aspects of the history of psychiatry. With respect to Cooper's analysis, I argued that the goal of classifying disorders as natural kinds was not - and should not be - the exclusive goal of psychiatric classification. Besides meeting epistemic goals, a classification manual like the DSM should be oriented towards achieving certain practical goals (e.g., the provision of a manual that promotes treatment), and I suggested that practical goals of psychiatric classification should sometimes be weighted more heavily than epistemic ones. With respect to Murphy’s analysis, I argued that his call for a causal and theoretical system of classification is strengthened by a historical outlook on psychiatric classification. Unless one assumes a naïve and ahistorical view of psychiatric classification, the DSM's atheoretical and descriptive approach cannot be seriously regarded as an adequate end-point for psychiatric

\footnotetext{
${ }^{71}$ APA, DSM-III, 7.

${ }^{72}$ See D. J. Kupfer, M. B. First, and D. A. Regier, “Introduction” in D. J. Kupfer, M. B. First, and D. A. Regier, A Research Agenda for DSM-V (Washington, DC: American Psychiatric Association, 2002), xviii-xix.

${ }^{73}$ D. A. Regier, W. E. Narrow, E. A. Kuhl, and D. J. Kupfer, “The Conceptual Development of DSM-V”, American Journal of Psychiatry 166 (2009), 648 -649, emphasis added.

${ }^{74}$ See S. E. Hyman, “Can Neuroscience be Integrated into the DSM-V?”, Nature Reviews Neuroscience 8 (2007), 725—732; Regier et al., “The Conceptual Development of DSM-V”, 649.
} 
classification. While DSM-III was certainly an advance in its historical context given the inadequacies of DSM-II, a shift to a theoretical and causal approach has strong potential - in the current historical context - to mark a further step of progress, from both an epistemic and pragmatic perspective.

A more general aim of the analysis of this paper was to illustrate the benefits of history for philosophy of psychiatry, and the virtues of historically-informed approaches to philosophy of science. Given the complex nature of psychiatric classification - which involves a mixture of epistemic and pragmatic considerations - a promising approach for addressing philosophical questions regarding classification is to examine the actual historical factors that led psychiatrists to adopt their current ideals of classification. The chief merit of this approach is that it can account for psychiatrists' own reasoning regarding issues of classification, while simultaneously considering the actual constraints that psychiatrists faced in addressing questions of how mental disorders should be classified. In this manner, a historical approach is capable of engaging with psychiatric practices and speaking to concerns that have been historically important in debates concerning the classification of mental disorders. In illustrating how an examination of the history of the DSM can qualify and expand upon existing philosophical analyses of psychiatric classification, I hope to have demonstrated the valuable methodological role that history can play in complementing philosophy of science analyses. ${ }^{75}$

\footnotetext{
${ }^{75}$ I owe thanks to Ian Hacking, Rachel Cooper, Rachel Ponce, Kristen Intemann, Kevin deLaplante, Darrel Regier, and an anonymous referee of this journal for helpful comments and suggestions on earlier versions of this paper. Research support from the Center of Excellence in the Arts and Humanities (CEAH) at Iowa State University is gratefully acknowledged.
} 